\title{
Variations in the dynamic response of structures founded on piles induced by obliquely incident SV waves *
}

\author{
C. Medina, G. M. Álamo, J. J. Aznárez, L. A. Padrón, O. Maeso \\ Instituto Universitario de Sistemas Inteligentes y Aplicaciones Numéricas en Ingeniería \\ (SIANI) Universidad de Las Palmas de Gran Canaria \\ Edificio Central del Parque Científico y Tecnológico \\ Campus Universitario de Tafira, 35017, Las Palmas de Gran Canaria, Spain \\ \{cristina.medina, guillermo.alamo, juanjose.aznarez, luis.padron, orlando.maeso\}@ulpgc.es
}

15 March 2019

\begin{abstract}
Although the seismic actions generally consist of a combination of waves, which propagates with an angle of incidence not necessarily vertical, the common practice when analysing the dynamic behaviour of pile groups is based on the assumption of vertically-incident wave fields. The aim of this paper is to analyse how the angle of incidence of SV waves affects the dynamic response of pile foundations and piled structures. A three-dimensional boundary element-finite element coupling formulation is used to compute impedances and kinematic interaction factors corresponding to several configurations of vertical pile groups embedded in an isotropic homogeneous linear viscoelastic half-space. These results, which are provided in ready-to-use dimensionless graphs, are used to determine the dynamic properties of slender and non-slender superstructures through a procedure based on a substructuring model. Results are expressed in terms of flexible-base period and damping as well as maximum shear force at the base of the structure. The relevance and main trends observed in the influence of the wavefront angle of incidence on the dynamic behaviour of the superstructure are inferred from the presented results. It is found that effective damping is significantly affected by the variations of the wave angle of incidence. Furthermore, it comes out that the vertical incidence is not always the worst-case scenario.
\end{abstract}

Keywords: pile foundations, seismic wave propagation, angle of incidence, soil-structure interaction, substructure model, kinematic interaction factors, effective damping

\section{Introduction}

When studying the seismic behaviour of foundations and structures, the system is usually assumed to be subjected to a vertically-incident wave field. Indeed, the seismic actions generally consist of a

${ }^{*}$ Draft of the paper originally published in Earquake Engineering \& Structural Dynamics 2019; 48:772-791. https://doi.org/10.1002/eqe.3160. This work is released with a Creative Commons Attribution Non-Commercial No derivatives License. 
combination of waves, which impinges on the ground surface with an angle of incidence not necessarily this parameter and often assuming that this hypothesis corresponds to the worst-case scenario.

The dynamic response of deep foundations under non-vertical excitation still demands further investigation. A pioneer work by Mamoon and Ahmad [1] analysed the effect of obliquely-incident $\mathrm{SH}, \mathrm{SV}$ and $\mathrm{P}$ waves on the seismic response of single piles expressed in terms of kinematic interaction factors. After that, other works (e.g. 2, 3, 4) addressed this problem for different configurations of pile groups subjected to obliquely-incident body waves or Rayleigh waves. Subsequently, Zarzalejos et al. [5] analysed how the type of seismic body wave and its angle of incidence affect bending moments at cap level of single piles and $3 \times 3$ pile groups. The relevant influence that the wave angle of incidence exerts on the dynamic response of pile foundations became apparent from the results of these investigations. However the influence of this parameter on the dynamic response of the superstructure has not received enough attention. Todorovska and Trifunac [6, 7] studied the dynamic behaviour of structures supported by embedded foundations and subjected to plane $\mathrm{P}$ and $\mathrm{SV}$ waves with several angles of incidence using a $2 \mathrm{D}$ model that considers a rigid foundation with semicircular shape. Moreover, Avilés et al. [8] analysed the influence of kinematic interaction effects on the relevant dynamic properties of structures supported by embedded and shallow foundations and subjected to obliquely incident $\mathrm{P}, \mathrm{SV}$ and Rayleigh waves.

Regarding pile-supported structures, Medina et al. [9] analysed the dynamic response of pilesupported buildings subjected to shear waves but only vertical incidence was considered in that work. Álamo et al. [10] employed a boundary element-finite element (BEM-FEM) model [11, 12] to obtain results for the dynamic response, in terms of maximum shear forces at the base of the structures, of a group of three structures supported on $3 \times 3$ pile groups and subjected to planar oblique shear waves. However, to the extent of the authors' knowledge, there are no parametric studies in the scientific literature examining the effects of the angle of incidence of seismic waves on the dynamic behaviour of structures supported on pile foundations consisting of a variable number of piles, with different embedment and spacing between them.

In order to contribute to filling this gap, this paper addresses an analysis of the dynamic response of slender and non-slender structures supported on pile groups subjected to SV obliquely-incident waves. The harmonic response of the soil-foundation system is obtained by making use of impedance functions and kinematic interaction factors computed through the BEM-FEM model mentioned above 11, 12, 13. Results corresponding to several configurations of vertical pile groups embedded in a homogeneous and viscoelastic half-space are provided in ready-to-use dimensionless graphs. These results allow not only analysing how the angle of incidence of the seismic waves affects the dynamic response of pile foundations but also that of the superstructures through a procedure based on a substructuring model in the frequency domain that takes into account both kinematic and inertial interaction effects [14. The variables employed in this case are flexible-base period and damping as well as maximum shear force at the base of the structure. Their values are computed, as functions of the angle of incidence of the seismic wave, for different configurations of foundations and superstructures. 


\section{Problem definition}

The dynamic response of one mode of vibration of a piled structure is studied in this paper through a three-degree-of-freedom (3DOF) system, as the one depicted in Figure 1 $\mathrm{b}$. This system is defined by the foundation horizontal displacement $u^{c}$ and rocking $\varphi^{c}$, together with the structural horizontal deflection $u$. In Figure 1 1 , displacements are written in terms of relative motions, $u_{r}^{c}=u^{c}-u_{g}$ and $\varphi_{r}^{c}=\varphi^{c}-\varphi_{g}$, where $u_{g}$ and $\varphi_{g}$ represent, respectively, the horizontal and rocking motions at the pile cap.

$(a)$

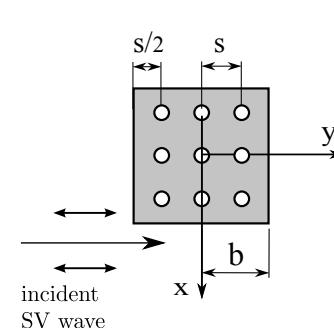

(b)

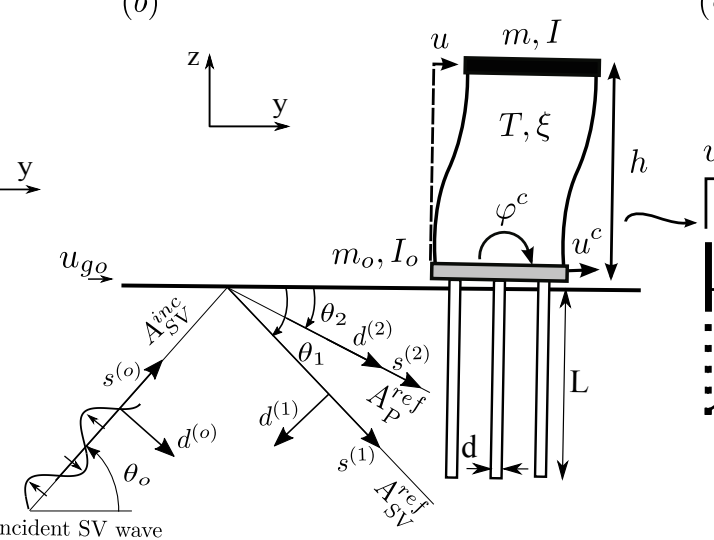

(c)

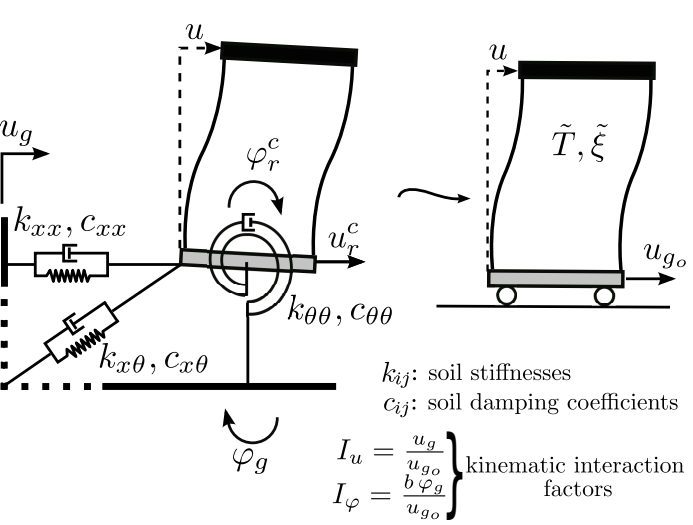

Figure 1: (a) Top view of the pile cap $(3 \times 3$ pile group configuration) (b) Problem definition (c) substructure model of a one-storey structure and $(\mathrm{d})$ equivalent single-degree-of-freedom oscillator.

The structure is considered to be supported on square regular groups of vertical piles embedded in a homogeneous, viscoelastic and isotropic halfspace. Pile heads are constrained by a rigid square pile cap $(2 b \times 2 b)$ which is assumed to be free of contact with the soil and whose mass $m_{o}$, moment of inertia $I_{o}$ and thickness are supposed to be negligible. All configurations of pile groups under study are symmetrical with respect to planes $x z$ and $y z$. Each one of them consists of a certain number of piles having all identical material properties as well as identical geometrical properties defined by length $L$ and sectional diameter $d$. The centre-to-centre spacing between adjacent piles is denoted by $s$ (see Figure 19).

The superstructure consists of a mass $m$ situated at the height $h$ of the resultant of the inertia forces for the mode of vibration under study and supported by massless and inextensible columns. The vibrating mass is assumed to be distributed over a square area and its moment of inertia is denoted by $I$. The structural dynamic behaviour, corresponding to fixed-base condition, is characterized by the structural fundamental period $T$ and its viscous damping ratio $\xi$.

\subsection{Dimensionless parameters}

In line with other authors [15, 16, 17, 18 and for the purpose of characterizing the soil-foundationstructure system, a set of dimensionless parameters, covering the mean features of SSI problems, has 
been used. These are the same parameters that were previously used in [9, 14]: (1) dimensionless fixed-base natural frequency of the structure $\lambda=\omega_{n} / \omega$; (2) fixed-base structure damping ratio $\xi$; (3) wave parameter $\sigma=c_{s} T / h$ that measures the soil-structure relative stiffness; (4) structural slenderness ratio $h / b ;(5)$ foundation-structure mass ratio $m_{o} / m$; (6) mass density ratio $\delta=m /\left(4 \rho_{s} b^{2} h\right)$ between structure and supporting soil; (7) Poisson's ratio $\nu_{s}$; and (8) damping ratio $\xi_{s}$ of the soil. A hysteretic damping model of the type $\mu_{s}=\operatorname{Re}\left[\mu_{s}\right]\left(1+2 \mathrm{i} \xi_{s}\right)$ is considered in this study for the soil material.

With respect to the pile foundation, the following dimensionless parameters are considered in this work: number of piles composing the pile group $(n \times n)$, pile spacing ratio $s / d$, embedment ratio $L / b$, pile slenderness ratio $L / d$, pile-soil Young's modulus ratio $E_{p} / E_{s}$ and soil-pile densities ratio $\rho_{s} / \rho_{p}$. The dimensionless excitation frequency is defined as $a_{o}=\omega b / c_{s}$, being $\omega$ the excitation circular frequency, $c_{s}=\sqrt{\mu_{s} / \rho_{s}}$ the speed of propagation of shear waves in the halfspace, and $\mu_{s}$ and $\rho_{s}$ the soil shear modulus of elasticity and mass density, respectively.

\subsection{Pile group configurations under investigation}

Different configurations of square $2 \times 2,3 \times 3$ and $4 \times 4$ vertical pile groups, according to the geometrical parameters defined in Figure 2, are analysed in the frequency range of interest for seismic loading. The dimensionless parameters corresponding to these configurations are listed in Table 1. Four different values are considered for the structural slenderness ratio $(h / b)$. Although a Poisson's ratio $\nu_{s}=0.08$ is not representative for typical soils, it has been included in this study as a limit value in order to explain some dynamic effects associated with the spatial character of the excitation and the kinematic response.

Table 1: Values for the dimensionless parameters in the cases under investigation

\begin{tabular}{ccccccccc}
\hline$\nu_{s}$ & $\xi_{s}$ & $E_{p} / E_{s}$ & $\rho_{s} / \rho_{p}$ & $\xi$ & $\delta$ & $1 / \sigma$ & $m_{o} / m$ & $h / b$ \\
\hline $0.08,0.2,1 / 3,0.4,0.45$ & 0.05 & $10^{3}$ & 0.7 & 0.05 & 0.15 & $0-0.5$ & 0 & $1,2,5,10$ \\
\hline
\end{tabular}

\section{$2.3 \quad$ Incident field}

The excitation (incident field) is considered to be a planar SV wave propagating through the halfspace with a generic direction contained in the $y z$ plane and defined by the angle of incidence $\theta_{o}$ measured from the horizontal (see Figure 1b). When non-vertically incident seismic SV body waves hit the free surface, SV waves are reflected back into the half-space together with $\mathrm{P}$ waves. This $\mathrm{P}$-waves are inhomogeneous waves propagating horizontally when the angle of incidence is smaller than the critical angle $\theta_{c r}$, which depends only on the Poisson's ratio, as shown in equation (11).

$$
\theta_{c r}=\arccos \left(\sqrt{\frac{1-2 \nu_{s}}{2\left(1-\nu_{s}\right)}}\right)
$$

Thus, the mechanism of propagation of the waves in the soil depends on whether the wave angle of incidence is greater or smaller than this critical angle, which will prove relevant when analysing the dynamic behaviour of the superstructure. 
The expressions for displacements vectors and amplitudes in function of the angle of incidence, for values over and below the critical angle, can be found (e.g.) in the classical texts of Elastodynamics written by Achenbach [19] or Eringen \& Suhubi [20] or more recently, in a summarized form, in Zarzalejos et al. [5].

\section{Methodology}

As mentioned before, the seismic response of the soil-foundation system is computed through a threedimensional frequency-domain BEM-FEM formulation previously developed [11, 12, 13, 5]. In this model, piles are modelled using monodimensional finite elements as Euler-Bernoulli beams, while soil is modelled using boundary elements as a linear, isotropic, homogeneous, viscoelastic medium. Welded boundary contact conditions at the pile-soil interfaces are assumed.

This BEM-FEM coupling model allows determining the dynamic response of the soil-foundation system in terms of translational $I_{u}\left(a_{o}, \theta_{o}\right)=u_{g} / u_{g_{o}}$ and rotational $I_{\varphi}\left(a_{o}, \theta_{o}\right)=\varphi_{g} b / u_{g_{o}}$ kinematic interaction factors, being $u_{g_{o}}$ the free-field motion at the surface. The impedance functions at each frequency $a_{o}$ can be also computed through this model. These impedance functions are usually written as $K_{i j}=k_{i j}+\mathrm{i} a_{o} c_{i j}$, where $k_{i j}$ and $c_{i j}$ are the frequency-dependent dynamic stiffness and damping coefficients, respectively.

Afterwards, impedances and kinematic interaction factors are used to analyse the 3DOF system dynamic response through a substructuring model in the frequency domain such as that represented in Figure 1k. This model consists of a building-cap structure supported on springs and dashpots representing the soil-foundation stiffness and damping in the horizontal $\left(k_{x x}, c_{x x}\right)$, rocking $\left(k_{\theta \theta}, c_{\theta \theta}\right)$ and cross-coupled horizontal-rocking $\left(k_{x \theta}, c_{x \theta}\right)$ vibration modes, respectively. The whole system is subjected to the horizontal $\left(u_{g}\right)$ and rocking $\left(\varphi_{g}\right)$ motions measured at the massless pile cap level when subjected to free-field motion at the surface $u_{g_{o}}$. A simple and accurate procedure [14] based on this substructuring model is used in order to determine the dynamic characteristics of an equivalent single-degree-of-freedom (SDOF) oscillator (see Figure 1 d d that reproduces, as accurately as possible, the response of the system under investigation (see Figure1 $\mathrm{c}$ ) within the range where the peak response occurs. This response is expressed in terms of $Q=\left|\omega_{n}^{2} u /\left(\omega^{2} u_{g_{o}}\right)\right|$, which represents the ratio of the shear force at the base of the structure to the effective earthquake force. The equivalent SDOF system can be defined by its damping ratio $\tilde{\xi}$ and its undamped natural period $\tilde{T}$.

The effective period $\tilde{T} / T$ and damping $\tilde{\xi}$ can be obtained from the following equations [14]:

$$
\begin{gathered}
\frac{\tilde{T}}{T}=\lambda \quad / \quad 1-\frac{1}{\lambda^{2}}-\frac{1}{\lambda^{2} \alpha_{x x}^{2}(\lambda)}-\frac{1}{\lambda^{2} \alpha_{\theta \theta}^{2}(\lambda)}=0 \\
\tilde{\xi}=\left|\left(I_{u}+\frac{h}{b} I_{\varphi}\right)^{-1}\left[\frac{\xi^{\prime}}{\tilde{\lambda}^{2}}+\frac{1}{\tilde{\lambda}^{2}}\left(\frac{\xi_{x x}}{\alpha_{x x}^{2}\left(1+\mathrm{i} 2 \xi_{x x}\right)}+\frac{\xi_{\theta \theta}}{\alpha_{\theta \theta}^{2}\left(1+\mathrm{i} 2 \xi_{\theta \theta}\right)}\right)\right]\right|
\end{gathered}
$$

where,

$$
\xi^{\prime}=\frac{\omega}{\omega_{n}} \xi
$$




$$
\begin{gathered}
\alpha_{x x}^{2}=\sigma^{2} \frac{1}{16 \pi^{2}} \frac{h}{b} \frac{1}{\delta} \tilde{k}_{x x} \\
\xi_{x x}=\frac{\tilde{c}_{x x}}{2 \tilde{k}_{x x}} \\
\alpha_{\theta \theta}^{2}=\sigma^{2} \frac{1}{16 \pi^{2}} \frac{h}{b} \frac{1}{\delta} \operatorname{Re}\left[\frac{b^{2}}{(h+D)^{2}} \tilde{K}_{\theta \theta_{D}}\right] \\
\xi_{\theta \theta}=\frac{\operatorname{Im}\left[\frac{b^{2}}{(h+D)^{2}} \tilde{K}_{\theta \theta_{D}}\right]}{2 \operatorname{Re}\left[\frac{b^{2}}{(h+D)^{2}} \tilde{K}_{\theta \theta_{D}}\right]}
\end{gathered}
$$

being $\tilde{K}_{x x}=K_{x x} /\left(\mu_{s} b\right)=\tilde{k}_{x x}+\mathrm{i} \tilde{c}_{x x}$ and

$$
\begin{gathered}
\tilde{K}_{\theta \theta_{D}}=\frac{1}{\mu_{s} b^{3}}\left(K_{\theta \theta}-\frac{K_{\theta x}^{2}}{K_{x x}}\right) \\
\frac{b^{2}}{(h+D)^{2}}=\left(\left(\frac{h}{b}\right)^{2}-2\left(\frac{h}{b}\right) \frac{\tilde{K}_{\theta x}}{\tilde{K}_{x x}}+\left(\frac{\tilde{K}_{\theta x}}{\tilde{K}_{x x}}\right)^{2}\right)^{-1}
\end{gathered}
$$

where $D=D(\omega)=-K_{x \theta} / K_{x x}$ represents the virtual depth of the point at which the soil-foundation interaction must be condensed to obtain a diagonal impedance matrix.

Finally, the maximum shear force at the base of the structure per effective earthquake force unit $Q_{m}$ is obtained as

$$
Q_{m}=\operatorname{Max}\left|\frac{1}{\left(\frac{\omega^{2}}{\omega_{n}^{2}}\left(\frac{\tilde{T}}{T}\right)^{2}-1\right)-\mathrm{i} 2 \tilde{\xi} \frac{\omega}{\omega_{n}} \frac{\tilde{T}}{T}}\right|
$$

The procedure followed to obtain these expressions for the effective period $\tilde{T} / T$ (equation (2)) and damping $\tilde{\xi}$ (equation (3)) and $Q_{m}$ (equation (11)) is thoroughly described in the aforementioned [14].

\section{Results}

This section presents the results obtained from the application of the methodology explained above to the analysis of the effects that the variation of the angle of incidence $\theta_{o}$ of SV waves has on the dynamic response of the configurations under investigation. 


\subsection{Kinematic interaction factors}

Figures 3 and 4 depict, respectively, the translational $I_{u}=u_{g} / u_{g_{o}}$ and the rotational $I_{\varphi}=b \varphi / u_{g_{o}}$ kinematic interaction factors corresponding to all the $3 \times 3$ pile group configurations under investigation for the following values of the angle of incidence: $\theta_{o}=30^{\circ}, 40^{\circ}, 50^{\circ}, 60^{\circ}, 70^{\circ} 80^{\circ}$ and $90^{\circ}$ (vertical incidence). Each column shows results for a different value of the soil Poisson's ratio $\nu_{s}$. Odd and even rows present, respectively, real and imaginary parts of the translational kinematic interaction factors corresponding to pile groups with different values of the pile slenderness ratio $L / d=7.5,15$ and 30 . The horizontal axis represents the dimensionless frequency.

As can be seen in Figures 3, the strongest filtering of the seismic excitation in terms of horizontal input motion occurs for the more horizontal of the considered angles of incidence $\left(\theta_{o}=30^{\circ}\right.$ and $\left.40^{\circ}\right)$. A remarkable filtering capacity is also noticed for angles of incidence more vertical than the critical angle while, on the contrary, for $\theta_{O}=50^{\circ}$, the horizontal input motion is amplified by the presence of the pile foundation.

On the other hand, as shown in Figure 4, the highest values of the rotational kinematic interaction factor $I_{\varphi}$ are reached for the those values considered for the angle of incidence that are below the critical angle $\left(\theta_{o}=30^{\circ}, 40^{\circ}\right.$ and $\left.50^{\circ}\right)$. This may be attributed in part to the way of presenting such information given that the horizontal free-field motion at the surface $u_{g_{o}}$ takes small values for these angles (see Figure 6, where the evolution of $u_{g_{o}} / A^{i n c}$ with $\theta_{o}$ is depicted, being $A^{i n c}$ the amplitude of the incident wave). It is worth noting that the responses for $\theta_{o}=30^{\circ}$ and $40^{\circ}$ are $180^{\circ}$ out of phase with respect to that corresponding to $\theta_{o}=50^{\circ}$ (opposite signs for one angle and the other). This effect plays an important role in the analysis of the structural response, as it will be shown subsequently, and occurs within a certain range of $\theta_{o}$. For the purpose of illustrating this fact, Figure 7 shows the evolution of the real part of the kinematic interaction factors $\left(\operatorname{Re}\left[I_{u}\right]\right.$ and $\left.\operatorname{Re}\left[I_{\varphi}\right]\right)$ with $\theta_{o}$ for different $3 \times 3$ pile groups configuration when $\omega d / c_{s}=0.1$. The range of $\theta_{o}$ in which $\operatorname{Re}\left[I_{u}\right]$ and $\operatorname{Re}\left[I_{\varphi}\right]$ have opposite signs is indicated with a shaded area. 


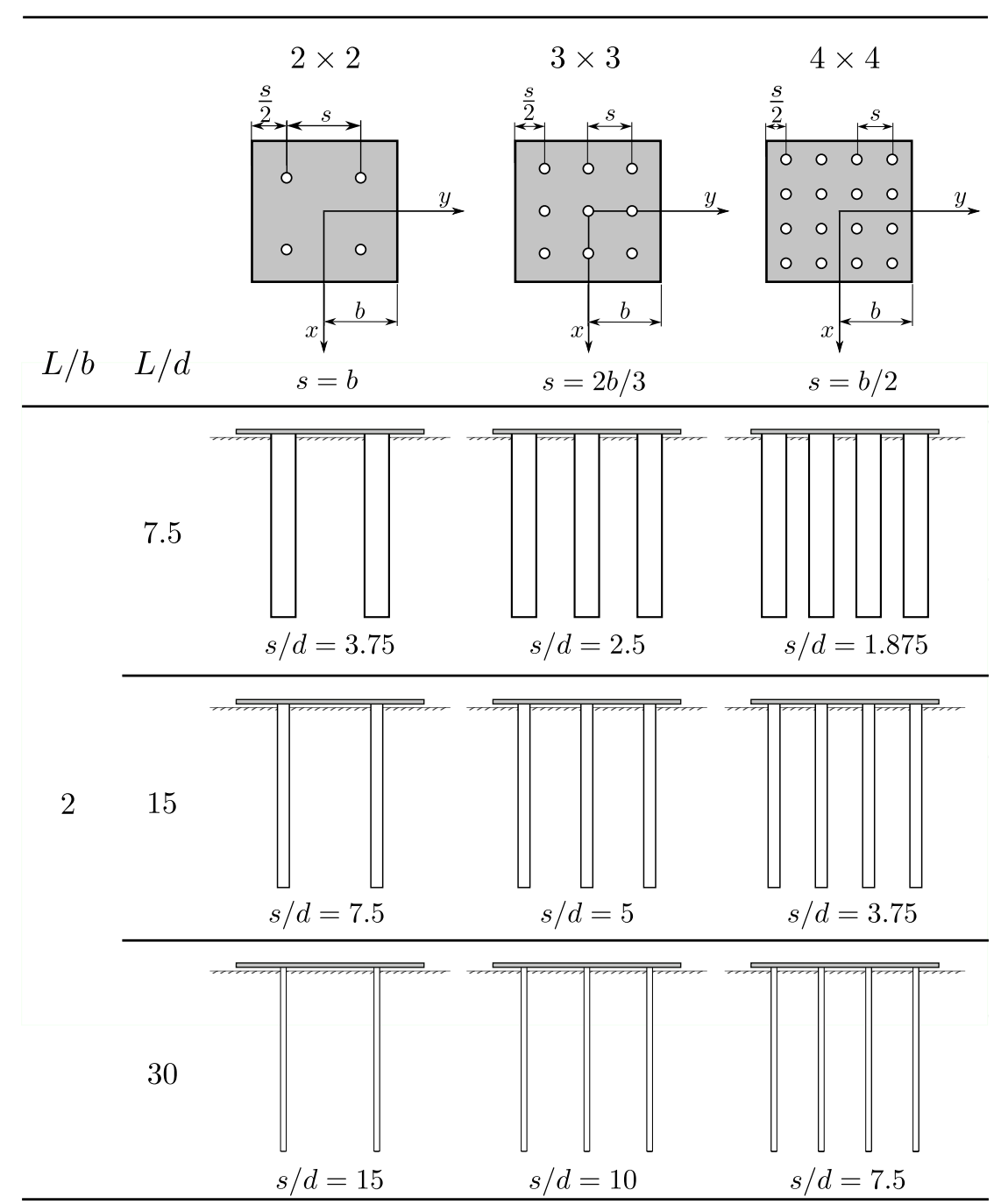

Figure 2: Geometric configuration of $2 \times 2,3 \times 3$ and $4 \times 4$ pile groups considered in this study. 

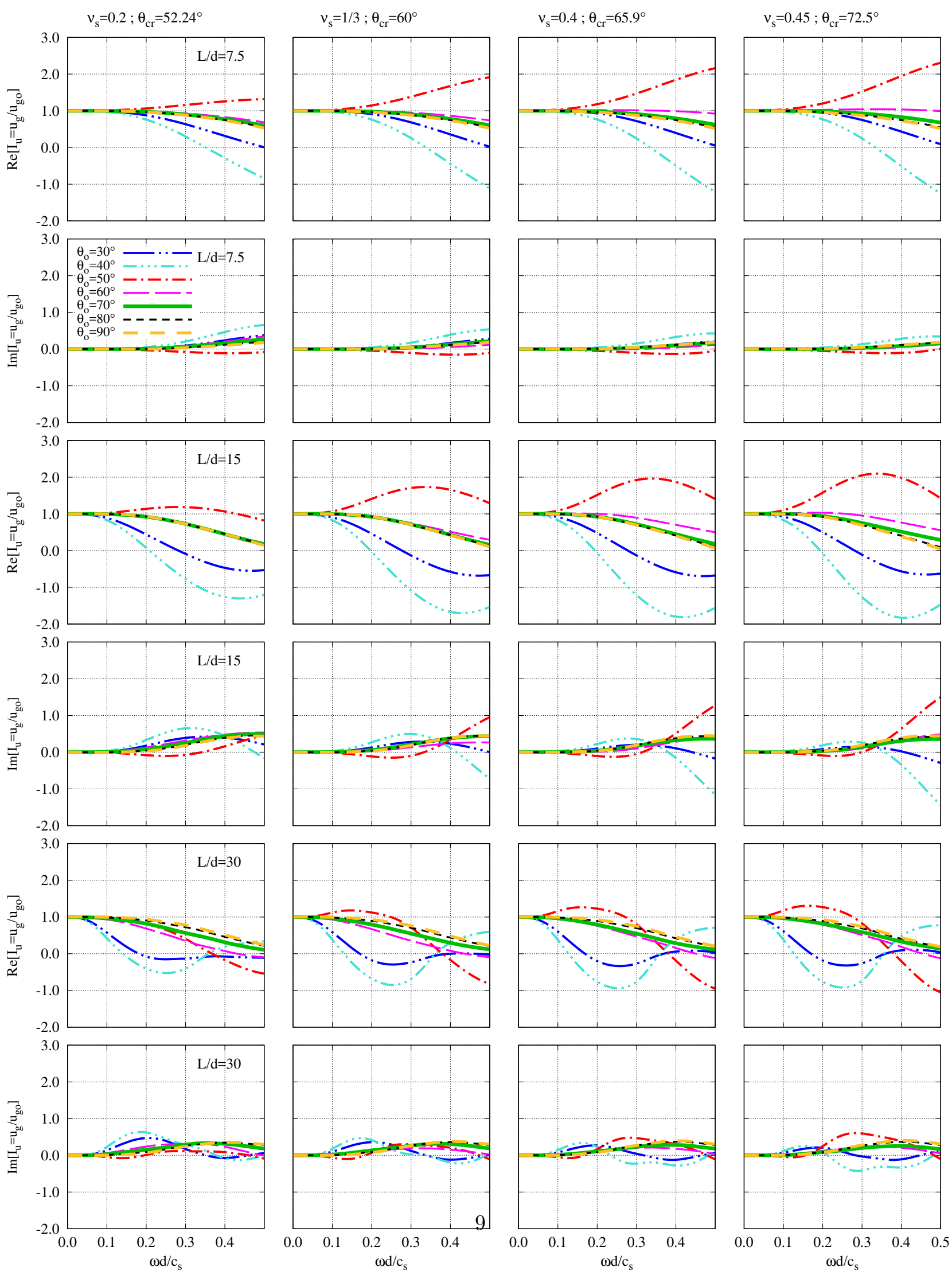

Figure 3: Translational kinematic interaction factor $I_{u}$ of $3 \times 3$ pile groups for different values of the angle of incidence $\theta_{o}$ of SV waves and several values of the Poisson's ratio $\nu_{s} . E_{p} / E_{s}=10^{3}$ and $\xi_{s}=0.05 . L / d(s / d)=7.5(2.5) 15(5), 30(10)$. 

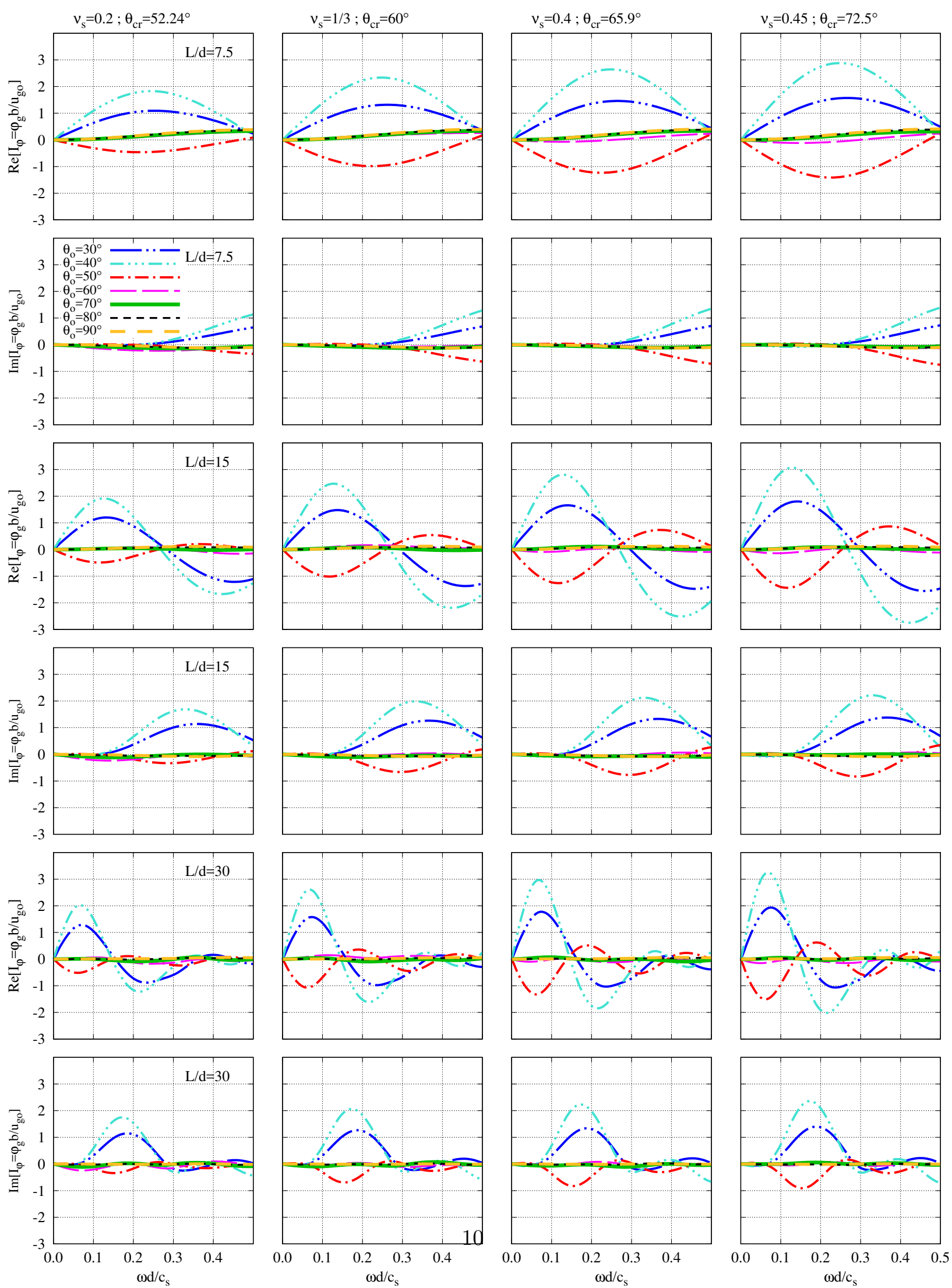

Figure 4: Rotational kinematic interaction factor $I_{\varphi}$ of $3 \times 3$ pile groups for different values of the angle of incidence $\theta_{o}$ of SV waves and several values of the Poisson's ratio $\nu_{s} . E_{p} / E_{s}=10^{3}$ and $\xi_{s}=0.05$. $L / d(s / d)=7.5(2.5) 15(5), 30(10)$. 

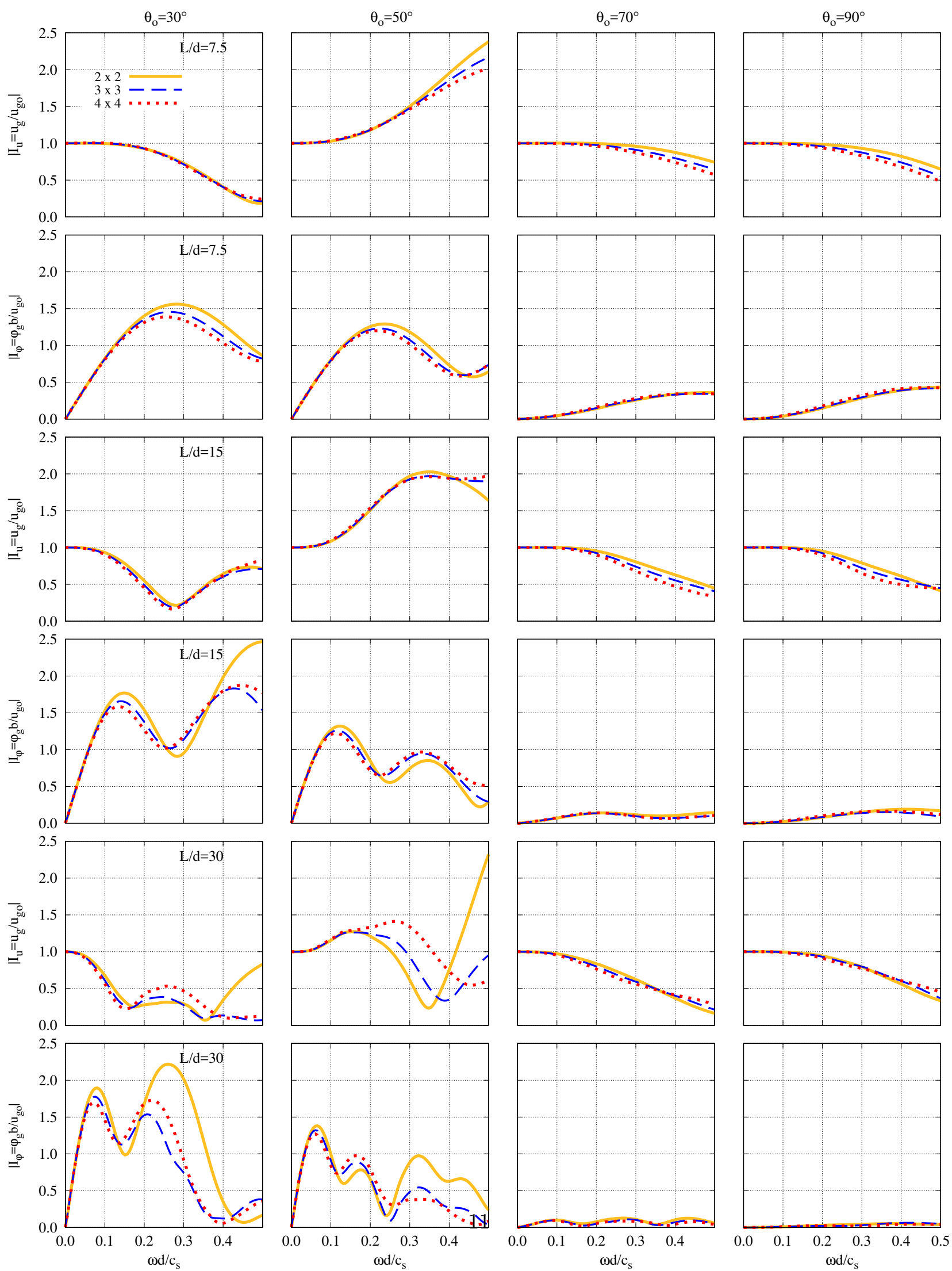

Figure 5: Kinematic interaction factors moduli $\left(\left|I_{u}\right|\right.$ and $\left.\left|I_{\varphi}\right|\right)$ of $2 \times 2,3 \times 3$ and $4 \times 4$ pile groups for different values of the angle of incidence $\theta_{o}$ of SV waves. $E_{p} / E_{s}=10^{3}, \xi_{s}=0.05$ and $\nu_{s}=0.4$. $L / d=7.5,15,30$. 

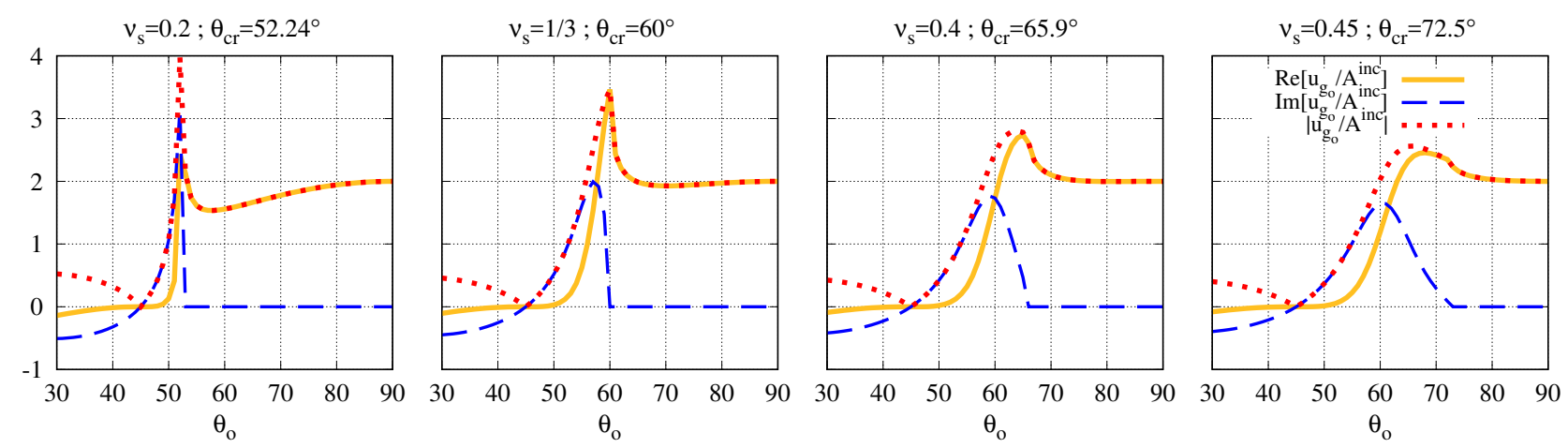

Figure 6: Variation of the free-field horizontal displacement $u_{g_{o}}$ with the angle of incidence $\theta_{o}$ for a SV incident wavefront considering different soils with $\nu_{s}=0.2,1 / 3,0.4$ and 0.45 .

Following the same format used in Figures 3 and 4 , Figure 5 depicts the moduli of the kinematic interaction factors corresponding to $2 \times 2$ (yellow line), $3 \times 3$ (blue line) and $4 \times 4$ (red line) pile groups with $L / d=7.5,15$ and 30 . It can be seen that the curves corresponding to configurations with different number of piles all follow the same trend. It is worth noting that for angles of incidence over the critical angle, the influence of the number of piles on the rotational kinematic interaction factors is negligible. A reduction of $I_{\varphi}$ is also observed as the pile slenderness ratio $L / d$ increases for angles of incidence over the critical angle.

The impedances of $2 \times 2,3 \times 3$ and $4 \times 4$ pile groups with $L / d=7.5,15$ and 30 as a function of the dimensionless frequency are extracted from Medina et al. [14]. These complex functions, together with those obtained for the kinematic interaction factors, are used to determine, in the following sections, the dynamic behaviour of the superstructure.

\subsection{Effective period}

As expected, the system effective period does not depend on the incident field. The variation of $\theta_{o}$ does not affect the system effective period because the kinematic interaction factors are not involved in the estimation of the value of $\tilde{T} / T$ corresponding to the SDOF equivalent system (see equation (2) ).

Figure 8 depicts the variation of the system effective period $(\tilde{T} / T)$ with the structure-soil relative stiffness $(1 / \sigma)$ for different configurations of pile groups. The left column illustrates how the increment of the undamped natural period $\tilde{T}$ with respect to the fixed-base natural period $T$ becomes more relevant, for instance, when the height of the structure increases. Note that this trend is reversed for non-slender structures when considering stiffer foundations. A similar effect can be observed in the central column when the diameter of the piles decreases (larger $L / d$ with constant $L / b$ ). The right column shows a reduction of the effective period for increasing number of piles. Results corresponding to the rest of the pile group configurations under study show the same trends but they are not included in this paper in pursuit of brevity. A thorough study analysing the influence of the main parameters involved in soil-structure interaction problems on the dynamic characteristics of structures supported on vertical pile groups was performed (for the first time to the authors' knowledge) by Medina et al. 


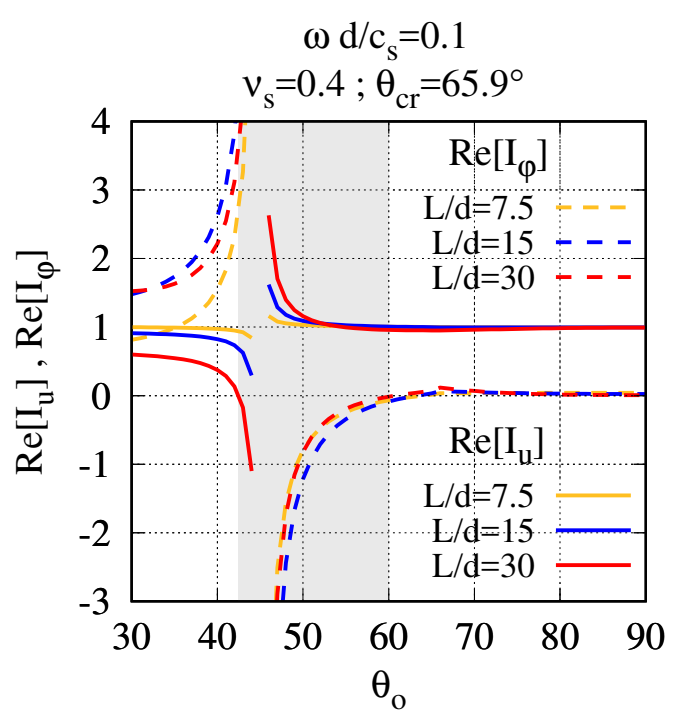

Figure 7: Real part of the kinematic interaction factors $I_{u}$ and $I_{\varphi}$ of $3 \times 3$ pile groups for different values of the angle of incidence $\theta_{o}$ of SV waves. $E_{p} / E_{s}=10^{3}, \xi_{s}=0.05$ and $\nu_{s}=0.4 . L / d(s / d)=$ $7.5(2.5) 15(5), 30(10) . \omega d / c_{s}=0.1$.

[14.

\subsection{Effective damping}

Figure 9 shows the system effective damping for the different configurations of $3 \times 3$ pile groups under investigation embedded in a soil such that $\nu_{s}=0.4$. Each row represents the results obtained for a pile group configuration with a different value of the pile slenderness ratio $L / d$. In turn, each column corresponds to a different value of the structural slenderness ratio $h / b=1,2,5$ and 10 . Results corresponding to several values of the angle of incidence $\left(\theta_{o}=30^{\circ}, 40^{\circ}, 50^{\circ}, 60^{\circ}, 70^{\circ}\right.$ and $90^{\circ}$ (vertical incidence)) are depicted. Results obtained involving only inertial interaction, which are computed without considering the kinematic effects of the incident field affecting the foundation, are also shown in this figure. As in Figure 8, the horizontal axis represents the inverse of the wave parameter $1 / \sigma$. It can be seen that for angles of incidence over the critical angle $\left(\theta_{o}>\theta_{c r}\right)$ the effective damping can be well approximated by that corresponding to vertical incidence. If kinematic interaction is neglected (inertial interaction curve), the computed effective dampings are higher than those obtained when kinematic interaction is taken into account for angles of incidence $\theta_{o}=90^{\circ}, 70^{\circ}, 40^{\circ}$ and $30^{\circ}$. This means that, for all these cases, neglecting kinematic interaction yields significantly non-conservative results, and should be avoided. It is worth noting that when $\theta_{O}=50^{\circ}$, a significant increase of $\tilde{\xi}$ occurs for certain values of $1 / \sigma$ which implies a great reduction of the maximum shear force $Q_{m}$ that reaches values close to zero. In order to illustrate this effect, Figure 10 presents $Q_{m}$ for the same pile group configurations analysed in Figure 9, which provide complementary results that are more practical from a engineering point of view. Greater values of $Q_{m}$ are obtained when the angle between the direction of propagation of the wavefront and the horizontal is $\theta_{o}=30^{\circ}$ or $40^{\circ}$ and minimum values 

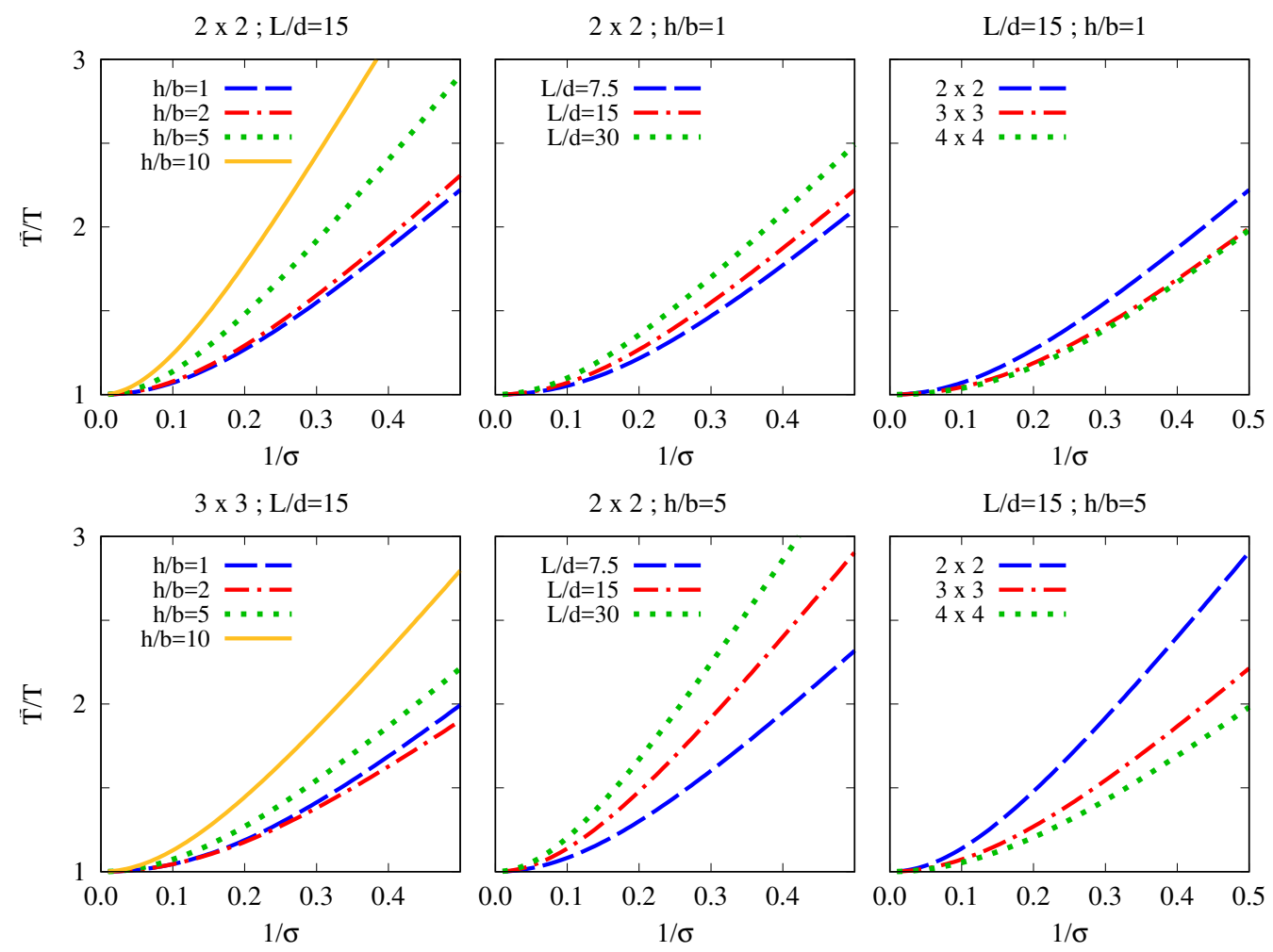

Figure 8: Influence of the structural slenderness ratio $h / b$ (left column), the pile slenderness ratio $L / d$ (central column) and the number of piles (right column) on the effective period $\tilde{T} / T . E_{p} / E_{s}=10^{3}$, $\xi_{s}=0.05$ and $\nu_{s}=0.4$.

of $Q_{m}$ are reached for $\theta_{o}=50^{\circ}$, in most cases. The influence of the foundation stiffness becomes more remarkable for angles of incidence below the critical angle. For slender structures the maximum shear force increases with $1 / \sigma$, reaching values beyond five times those corresponding to vertical incidence for the case in which $h / b=10$. However, for short or medium-height buildings $(h / b<5), Q_{m}$ reaches its maximum for values of the wave parameter such that $1 / \sigma<0.2$.

This behaviour of the structural dynamic response when $\theta_{o}=50^{\circ}$ is related with the aforementioned change of sign observed in the rotational kinematic interaction factor $I_{\varphi}$ and it can be explained from the expression of the system effective damping $\tilde{\xi}$ written in equation (3). It can be inferred that, for a certain configuration, the maximum value of $\tilde{\xi}$ is achieved when

$$
I_{u}(\tilde{T} / T) \approx-\frac{h}{b} I_{\varphi}(\tilde{T} / T)
$$

which is to say

$$
\frac{u_{g}}{b \varphi_{g}} \approx-\frac{h}{b}
$$



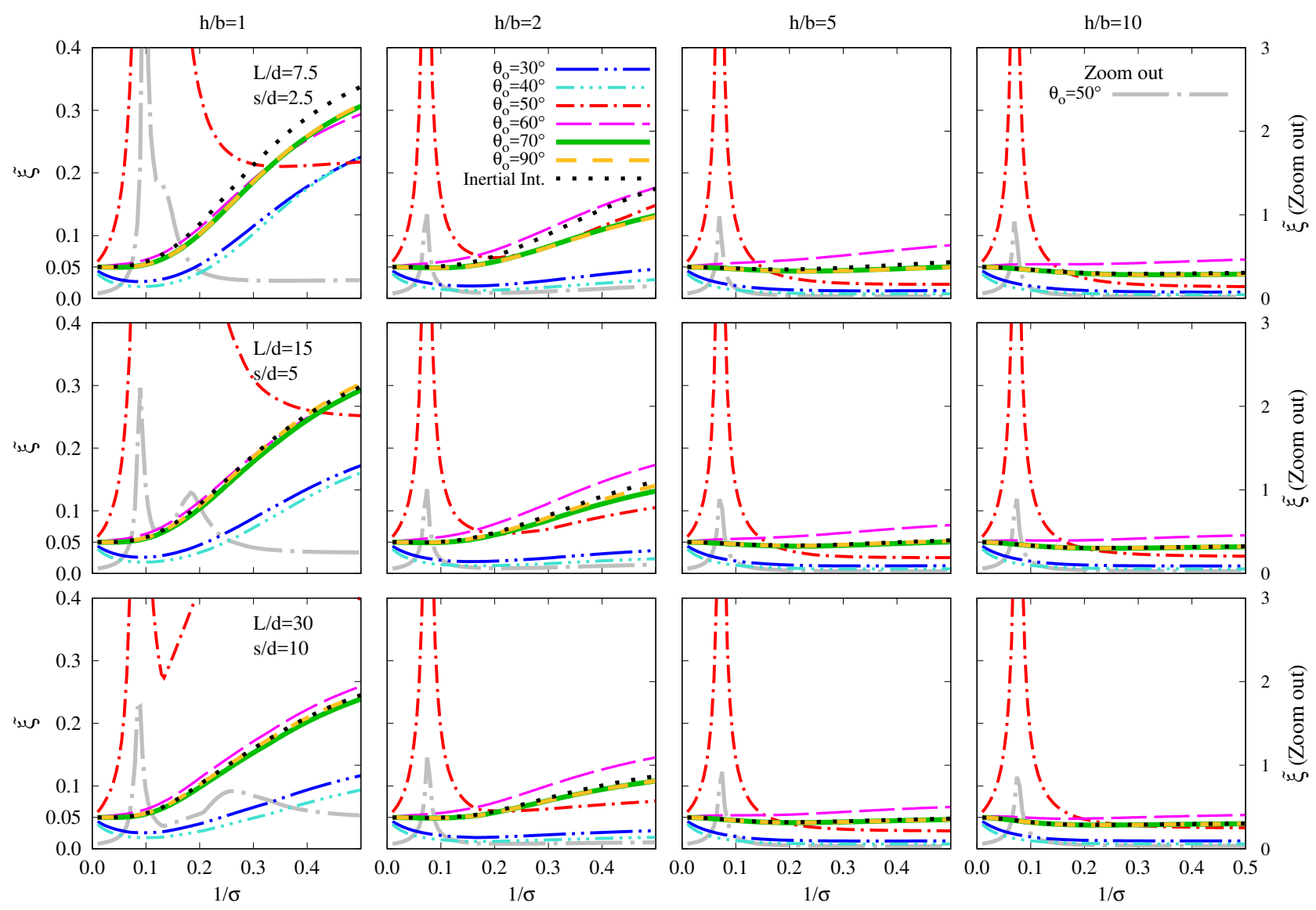

Figure 9: Effective damping $\tilde{\xi}$ for different $3 \times 3$ pile groups. $E_{p} / E_{s}=10^{3}, \xi_{s}=0.05$ and $\nu_{s}=0.4$ $\left(\theta_{c r}=65.9^{\circ}\right) . L / d(s / d)=7.5(2.5) 15(5), 30(10)$. Grey line to be read on the right axis provide a zoomed out view.

Defining $r=u_{g} / \varphi_{g}$ as the vertical distance between the free surface and the system center of rotation (being $r>0$ when the system center of rotation is under the free surface), it can be inferred that when $r$ reaches values close to the height of the structure $(-r \approx h)$, the behaviour of the superstructure closely resembles that of a rigid solid whose center of rotation is located in the centre of the slab. Consequently, the structural horizontal deflection $u$ and, in turn, the maximum shear force at the base of the structure $Q_{m}$, experience a significant reduction which implies a considerable increase of the system effective damping $\tilde{\xi}$. For the purpose of illustrating this effect, Figure 11 shows in each graphical area three curves superimposed that correspond, respectively, to $r / b\left(a_{o}\left(Q_{m}\right)\right)=$ $\operatorname{Re}\left[I_{u}\right] / \operatorname{Re}\left[I_{\varphi}\right]$, the structural slenderness ratio $h / b$ and the system effective damping $\tilde{\xi}$ for slender and non-slender structures supported on a $2 \times 2$ pile group subjected to SV waves being $\theta_{o}=50^{\circ} . a_{o}\left(Q_{m}\right)$ is the value that takes the dimensionless frequency when the maximum shear force at the base of the structure $Q_{m}$ occurs. Only the real parts of the kinematic interaction factors, $\operatorname{Re}\left[I_{u}\right]$ and $\operatorname{Re}\left[I_{\varphi}\right]$, have been considered because their imaginary parts are negligible in both cases. As for previous Figures, the 

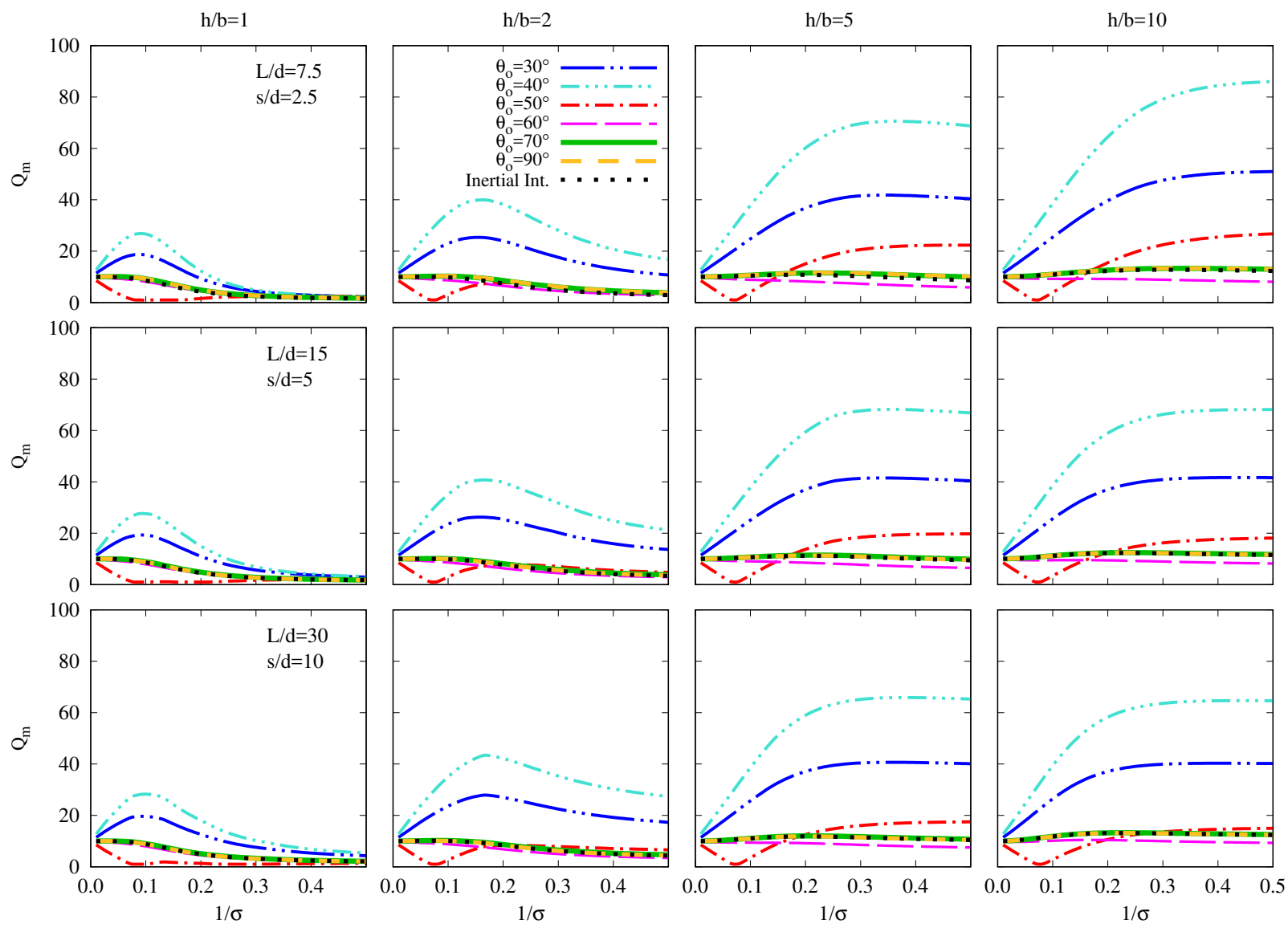

Figure 10: Maximum structural response value $Q_{m}$ for different $3 \times 3$ pile groups. $E_{p} / E_{s}=10^{3}$, $\xi_{s}=0.05$ and $\nu_{s}=0.4\left(\theta_{c r}=65.9^{\circ}\right) . L / d(s / d)=7.5(2.5) 15(5), 30(10)$.

horizontal axis represents the inverse of the wave parameter $1 / \sigma$ and each column depicts results for a different structural slenderness ratio $h / b$. In turn, each row presents results for different values of the Poisson's ratio $\nu_{s}$. In this figure, it can be clearly seen that the maximum values of the system effective damping $\tilde{\xi}$ are reached for those values of the wave parameter at which the curve corresponding to $-r / b$ intersects that of $h / b$, as occurs for $\nu_{s}=0.4$ and $\nu_{s}=1 / 3$.

The bottom row in Figure 11 illustrates that when $\nu_{s}=0.08,-r / b$ and $h / b$ functions do never intersect. In this case, the system effective damping $\tilde{\xi}$ varies with a monotonous trend as the wave parameter decreases. When $\nu_{s}=0.4$ or $\nu_{s}=1 / 3$, the angle between the direction of propagation of the wavefront and the horizontal $\left(\theta_{o}=50^{\circ}\right)$ is below the critical angle $\theta_{c r}$, while for $\nu_{s}=0.08$ (not a representative for typical soils and only used in order to explain this effect) the angle of incidence of the SV wave is over the critical angle.

At this stage, it is worth determining the range of values of the angle of incidence $\theta_{o}$ of SV waves for which the superstructure rotates almost as a rigid solid around the centre of the slab that remains practically at the same place. For this purpose, the problem has been analysed for a $2 \times 2$ pile group 
subjected to SV waves with angles of incidence between $\theta_{o}=40^{\circ}$ and $\theta_{o}=70^{\circ}$ with an increment of $1^{\circ}$. Figure 12 presents the maximum effective damping values obtained for each soil, angle of incidence and slenderness ratio, together with the values of the other relevant functions for the configuration at which that happens. Each column corresponds to a different value of the soil Poisson's ratio $\nu_{s}$ and, consequently, to a different value of the critical angle $\theta_{c r}$ which has been depicted in each graphical area with a solid vertical black line. The first and the second rows show the values of the kinematic interaction factors real parts, $\operatorname{Re}\left[I_{u}\right]$ and $\operatorname{Re}\left[I_{\varphi}\right]$, for the dimensionless frequency $a_{o}(\operatorname{Max}[\tilde{\xi}])$ at which the maximum value of the system effective damping is reached. The third row of this figure depicts the ratio of the distance between the pile cap centre and the system center of rotation to the foundation halfwidth $(-r / b)$. With the aim of facilitating the interpretation of the presented results, the different values of the structural slenderness ratio are represented, in each graphical area of the third row, with horizontal lines of the corresponding colours. The fourth row shows the maximum value reached for the system effective damping $\operatorname{Max}[\tilde{\xi}]$ within the range of values considered for the inverse of the wave parameter $1 / \sigma$. For the purpose of providing the reader with complementary information, the bottom row depicts the values of the inverse of the wave parameter $1 / \sigma$ for which the maximum value of $\tilde{\xi}$ is reached for each value of the angle of incidence $\theta_{o}$.

Figure 12 aims at helping the reader to understand the aforementioned effect (a great increase of the system effective damping $\tilde{\xi}$ associated with those values of the angle of incidence $\theta_{o}$ for which $\left(u_{g}\right)$ and $\left(\varphi_{g}\right)$ are out of phase and the ratio $-r / b$ is close to the structural slenderness ratio $\left.h / b\right)$ and to delimit the range of $\theta_{o}$ (indicated with a shaded area) in which this effect occurs for each type of soil. It can be seen that this effect occurs for $45^{\circ}<\theta_{o}<\theta_{c r}$. It is worth noting that this range widens for more incompressible soils. The same type of graph is presented in Figure 13 for a $3 \times 3$ pile group yielding the same conclusions. In order to enhance the reader understanding of Figures 12 and 13 , the evolution of the location of the system center of rotation within the considered range of $\theta_{o}$ and its consequences on the structural behaviour is illustrated in Figure 14. All plots in Figures 12 and 13 show the same four zones: a) for $\theta_{o}<45^{\circ}$, the system center of rotation is placed at $0<-r<h$, and the effective damping is low; b) for $45^{\circ}<\theta_{o}<\theta_{\alpha}$ (see Figure 14), the height of the system center of rotation is $r=-h$, which yields a very low effective earthquake shear force $Q$ (a high effective damping); c) for $\theta_{\alpha}<\theta_{o}<\theta_{\beta}$, the system center of ration is placed at $-r>h$, and the effective damping is low again; and d) for $\theta_{o}>\theta_{\beta}$, the system center of rotation is placed below the free surface and the effective damping is again low. The values of $\theta_{\alpha}$ and $\theta_{\beta}$ depend on the specific problem. 

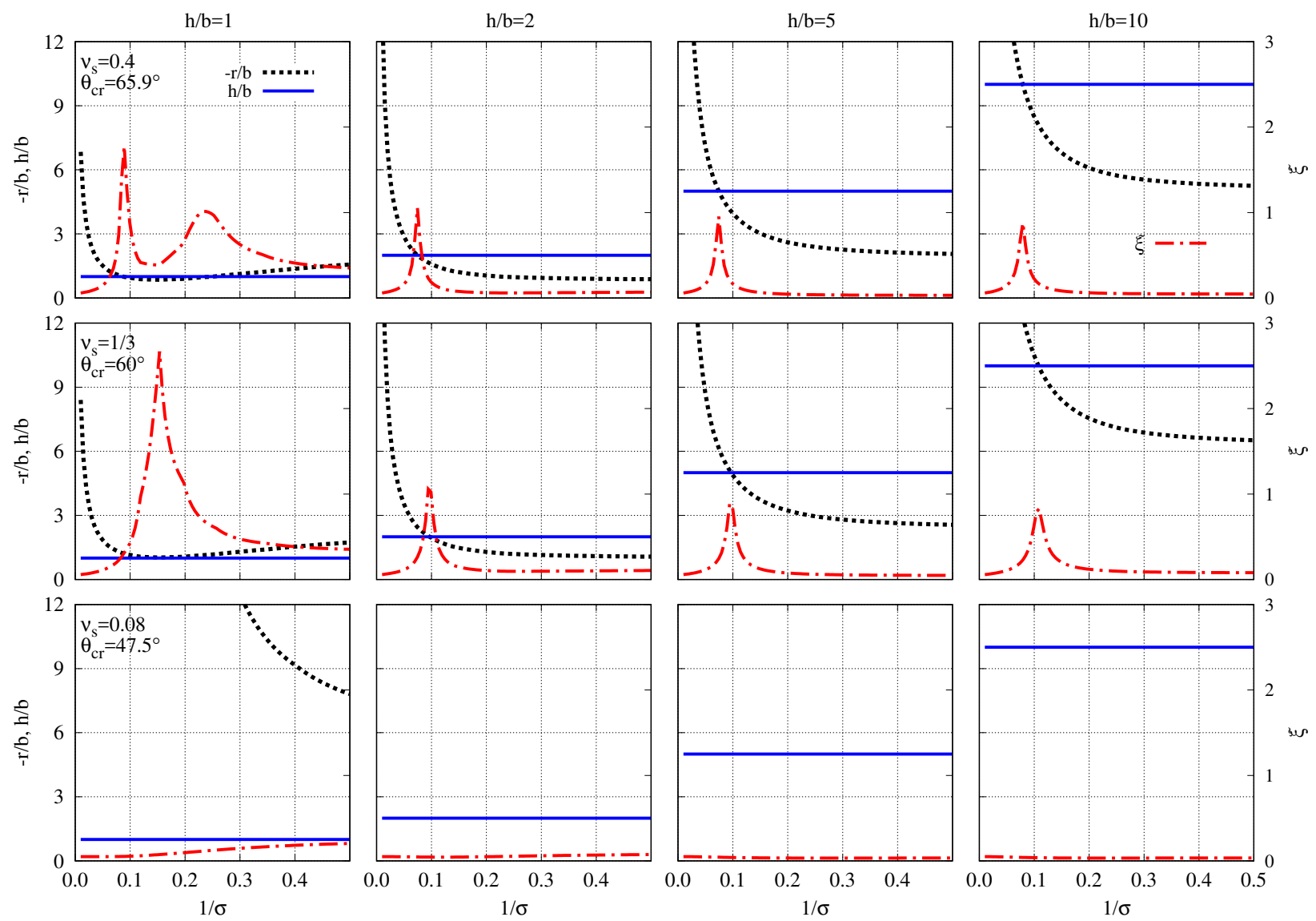

Figure 11: System center of rotation $r / b\left(a_{o}\left(Q_{m}\right)\right)$ and effective damping $\tilde{\xi}$ corresponding to different structures supported on a $2 \times 2$ pile group with $s / d=7.5, L / b=2$ y $E_{p} / E_{s}=10^{3}$ and subjected to $\mathrm{SV}$ waves with angle of incidence $\theta_{o}=50^{\circ}$. Influence of the soil Poisson's ratio. 

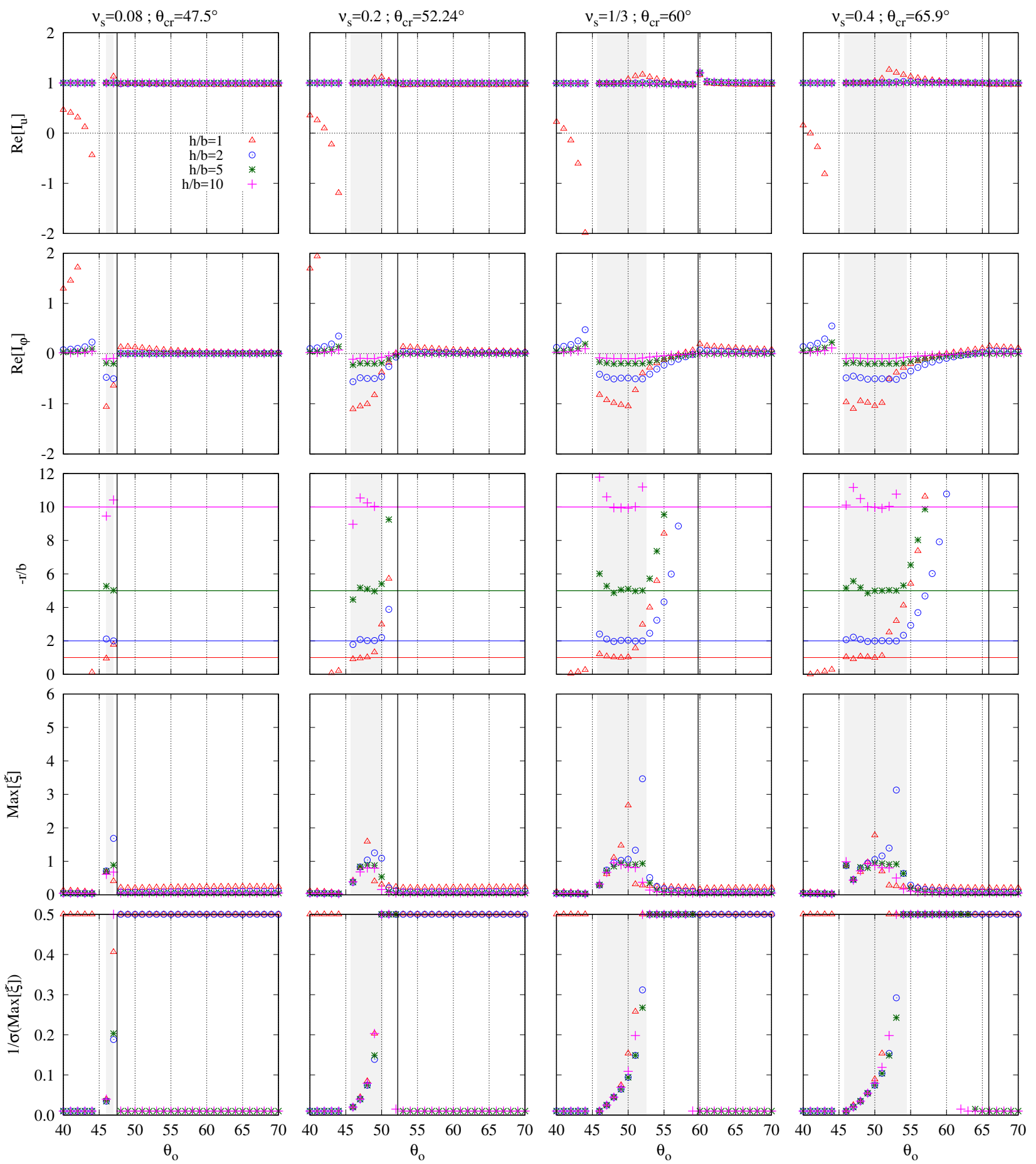

Figure 12: Maximum effective damping $(\operatorname{Max}[\tilde{\xi}])$ for structures with different slenderness ratios $(h / b)$ supported on a $2 \times 2$ pile group with $s / d=7.5, L / d=15, L / b=2$ and $E_{p} / E_{s}=10^{3}$. Kinematic interaction factors $\left(I_{u}=u_{g} / u_{g_{o}}\right.$ and $\left.I_{\varphi}=b \varphi_{g} / u_{g_{o}}\right)$ of the foundation for the dimensionless frequency $a_{o}$ at which the structural response $Q$ reaches its maximum value $\left(Q_{m}\right)$. System center of rotation $r / b\left(a_{o}\left(Q_{m}\right)\right)$. Values of the wave parameter $\sigma$ for 1 ryhich the maximum value of the effective damping is reached. 

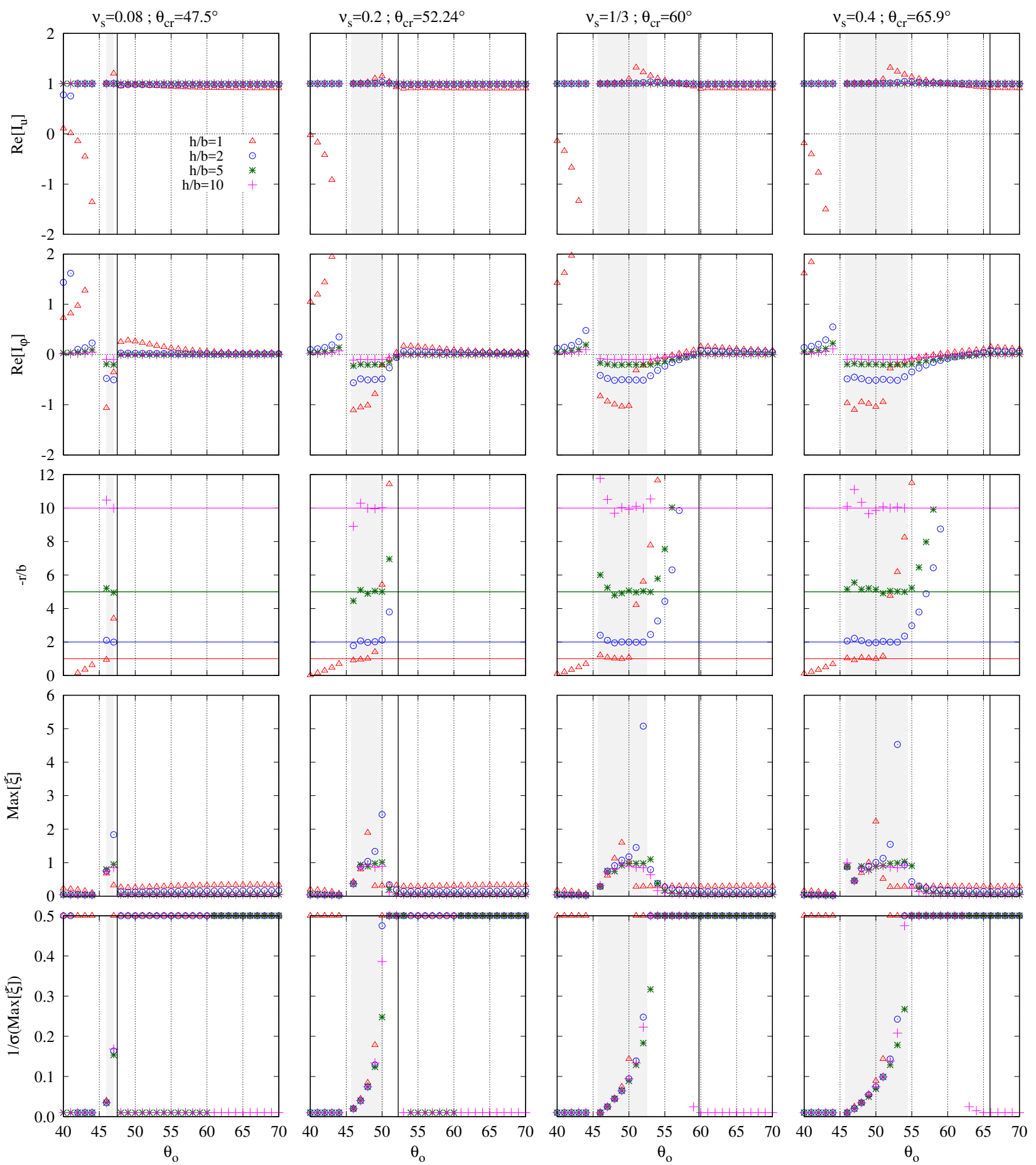

Figure 13: Maximum effective damping $(\operatorname{Max}[\tilde{\xi}])$ for structures with different slenderness ratios $(h / b)$ supported on a $3 \times 3$ pile group with $s / d=5, L / d=15, L / b=2$ and $E_{p} / E_{s}=10^{3}$. Kinematic interaction factors $\left(I_{u}=u_{g} / u_{g_{o}}\right.$ and $\left.I_{\varphi}=b \varphi_{g} / u_{g_{o}}\right)$ of the foundation for the dimensionless frequency $a_{o}$ at which the structural response $Q$ reaches its maximum value $\left(Q_{m}\right)$. System center of rotation $r / b\left(a_{o}\left(Q_{m}\right)\right)$. Values of the wave parameter $\sigma$ for 20 hich the maximum value of the effective damping is reached. 

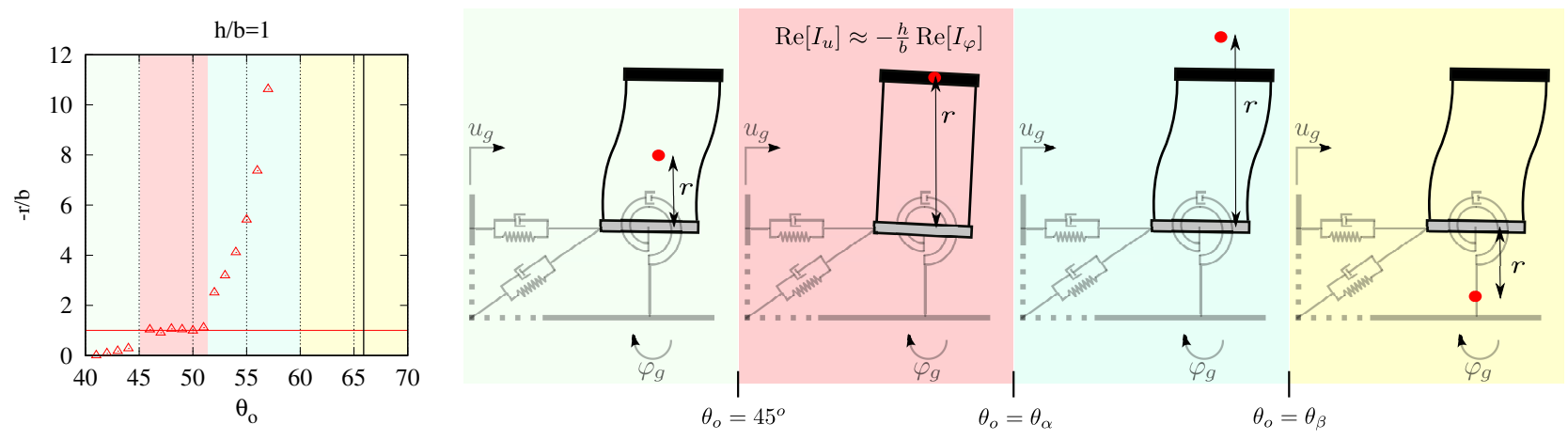

Figure 14: Influence of the wavefront angle of incidence $\theta_{0}$ on the location of the system center of rotation.

For the purpose of illustrating how the wavefront angle of incidence $\theta_{o}$ and the dimensionless frequency expressed as $\omega d / c_{s}$ affect the location of the system center of rotation, Figure 15] depicts results in terms of $-r / b$ for $2 \times 2$ pile group configurations with different values of the pile slenderness ratio $L / d(s / d)=7.5(3.75), 15(7.5)$ and $30(15)$. Those points in which the system center of rotation is located at the structural height $(-r / b=h / b)$ are represented with different colours corresponding to the superstructures considered in this study ( $h / b=1$ (red), 2 (blue), 5 (green) and 10 (cyan)). The projection of these points on the horizontal plane is represented in order to facilitate the comprehension of the results. Note that the range in which the structure can behave almost as a rigid solid $(-r \approx h)$ whose center of rotation is located in the centre of the slab widens as the foundation stiffness decreases (i.e. greater values of $L / d$ ).
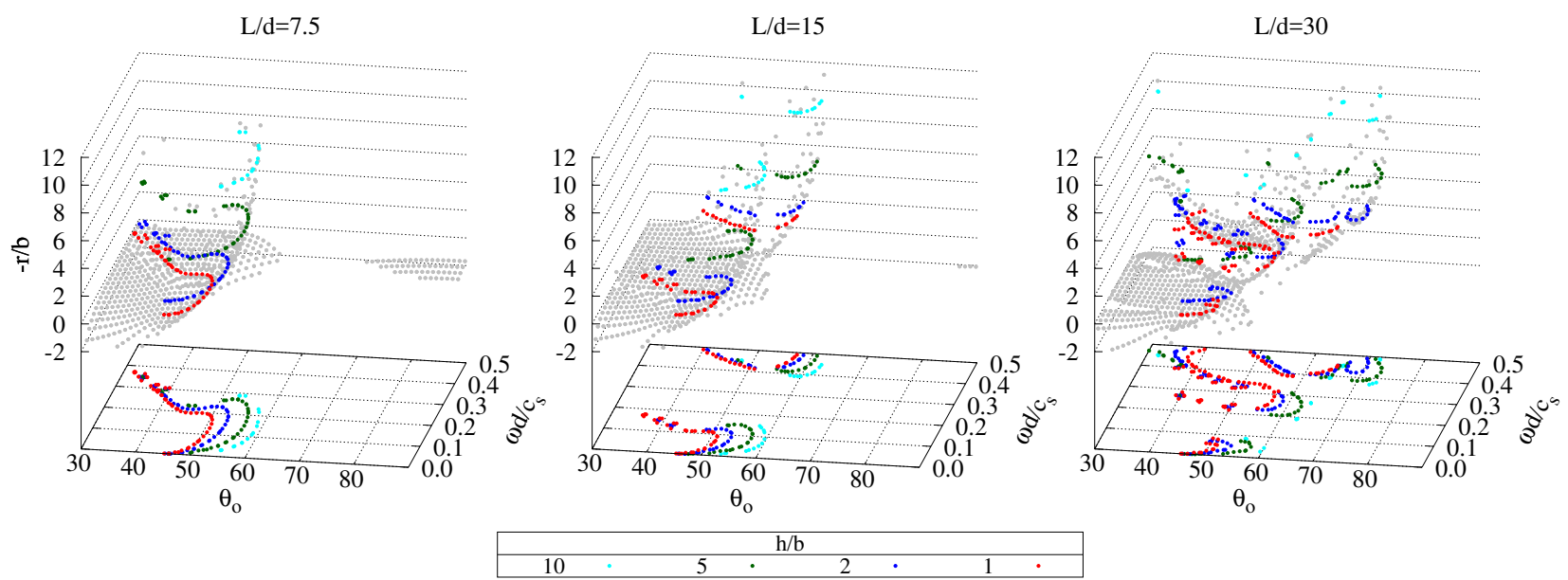

Figure 15: Influence of the wavefront angle of incidence $\theta_{o}$ and the dimensionless frequency $\omega d / c_{s}$ on the location of the system center of rotation of $2 \times 2$ pile group configurations for $\nu_{s}=0.4$. 


\section{Conclusions}

This paper addresses an analysis of the influence of the direction of propagation of SV waves on the dynamic response of pile foundations and piled structures. A BEM-FEM coupling formulation is used in this work to compute impedance functions and kinematic interaction factors of several pile group configurations considering different values of the angle of incidence. Subsequently, a simple and accurate substructuring procedure is used to determine the dynamic characteristics of a SDOF equivalent system that reproduces the dynamic response of the interacting system within the range where the peak response occurs. This dynamic response is expressed in terms of shear force at the base of the structure for effective earthquake force $Q$.

The dynamic behaviour of structures with different slenderness ratios $(h / b=1,2,5$ and 10) supported by $2 \times 2,3 \times 3$ and $4 \times 4$ pile groups and subjected to SV waves with several angles of incidence $\left(\theta_{o}\right)$ is analysed in this work. The main conclusions inferred from these results are summarised below:

- The results obtained for the translational kinematic interaction factor $I_{u}$ show that the strongest filtering of the seismic excitation can be observed when the angle between the direction of propagation of the wavefront and the horizontal is $\theta_{o}=30^{\circ}$ or $40^{\circ}$, among the cases studied herein. A noticeable filtering capacity is also shown for angles of incidence more vertical than the critical angle. On the contrary, when $\theta_{o}=50^{\circ}$, the ability of the foundation to filter SV waves decreases significantly.

- The highest values of the rotational kinematic interaction factor $I_{\varphi}$ are reached for angles of incidence below the critical angle $\left(\theta_{o}=30^{\circ}, 40^{\circ}\right.$ and $\left.\theta_{o}=50^{\circ}\right)$.

- The influence of the foundation stiffness on the maximum structural response $Q_{m}$ becomes more noticeable for angles of incidence below the critical angle.

- It is shown that the hypothesis usually adopted, vertical incidence, does not always corresponds to the worst-case scenario.

- When kinematic interaction is neglected, the computed effective dampings are over those obtained when kinematic interaction is considered for angles of incidence $\theta_{o}=90^{\circ}, 70^{\circ}, 40^{\circ}$ and $30^{\circ}$. Therefore, for all these cases, neglecting kinematic interaction yields significantly nonconservative results, and should be avoided.

- For slender structures the maximum structural response increases with relative structure-soil stiffness for shallow incidence $\left(\theta_{o}=30^{\circ}\right.$ or $\left.40^{\circ}\right)$, reaching values beyond five times those corresponding to vertical incidence for the case in which $h / b=10$. However, for short or mediumheight buildings $(h / b<5), Q_{m}$ reaches its maximum for values of the wave parameter such that $1 / \sigma<0.2$.

- Very low structural responses $Q_{m}$ can be reached when the angle of incidence is below the critical angle, but above $45^{\circ}$. This effect has not been mentioned by other authors in previous studies and it can be explained attending to the fact that the horizontal $\left(u_{g}\right)$ and rocking $\left(\varphi_{g}\right)$ motions measured at the pile cap level are out of phase and the instantaneous centre of rotation 
of the structure is close to the centre of the vibrating mass $-r / b \approx h / b$. This implies that the superstructure rotates almost as a rigid solid and the structural deflection is close to zero $(u \approx 0)$. The more incompressible the soil, the larger the range of incident angles for which very low maximum structural responses arise. It also has been checked that the influence of the foundation-structure mass ratio $m_{o} / m$ on this effect (and the general response) is negligible for all the cases under investigation.

\section{Acknowledgements}

This work was supported by the Subdirección General de Proyectos de Investigación of the Ministerio de Economía y Competitividad (MINECO) of Spain and FEDER through research project BIA201457640-R. G.M. Álamo is a recipient of FPU research fellowship FPU14/06115 from the Ministerio de Educación, Cultura y Deporte of Spain. The authors are grateful for this support.

\section{References}

[1] S. M. Mamoon, S. Ahmad, Seismic response of piles to obliquely incident SH, SV and P waves, Journal of Geotechnical Engineering 116 (1990) 186-204.

[2] S. M. Mamoon, P. K. Banerjee, Response of piles and pile groups to travelling SH-waves, Earthquake Engineering \& Structural Dynamics 19 (1990) 597-610.

[3] N. Makris, D. Badoni, Seismic response of pile groups under oblique-shear and Rayleigh waves, Earthquake Engineering \& Structural Dynamics 24(4) (1995) 517-532.

[4] A. M. Kaynia, M. Novak, Response of pile foundations to Rayleigh waves and obliquely incident body waves, Earthquake Engineering \& Structural Dynamics 21 (1992) 303-318.

[5] J. M. Zarzalejos, J. J. Aznárez, L. A. Padrón, O. Maeso, Influences of type of wave and angle of incidence on seismic bending moments in pile foundations, Earthquake Engineering \& Structural Dynamics 43 (2014) 41-59.

[6] M. I. Todorovska, M. D. Trifunac. Analytical model for in plane building-foundation-soil interaction: incident, Report No. CE 90-01, Department of Civil Engineering, University of Southern California, Los Angeles, California, 1990.

[7] M. I. Todorovska, M. D. Trifunac, The system damping, the system frequency and the system response peak amplitudes during in-plane building-soil interaction, Earthquake Engineering \& Structural Dynamics 21(2) (1992) 127-144.

[8] J. Avilés, M. Suárez, F. J. Sánchez-Sesma, Effects of wave passage on the relevant dynamic properties of structures with flexible foundation, Earthquake Engineering \& Structural Dynamics 31 (2002) 139-159. 
[9] C. Medina, L. A. Padrón, J. J. Aznárez, O. Maeso, Influence of pile inclination angle on the dynamic properties and seismic response of piled structures, Soil Dynamics and Earthquake Engineering 69 (2015) 196-206.

[10] G. M. Álamo, L. A. Padrón, J. J. Aznárez, O. Maeso, Structure-soil-structure interaction effects on the dynamic response of piled structures under obliquely incident seismic shear waves, Soil Dynamics and Earthquake Engineering 78 (2015) 142-153.

[11] L. A. Padrón, J. J. Aznárez, O. Maeso, BEM-FEM coupling model for the dynamic analysis of piles and pile groups, Engineering Analysis with Boundary Elements 31(6) (2007) 473-484.

[12] L. A. Padrón, J. J. Aznárez, O. Maeso, 3-D boundary element-finite element method for the dynamic analysis of piled buildings, Engineering Analysis with Boundary Elements 35(3) (2011) 465-477.

[13] C. Medina, L. A. Padrón, J. J. Aznárez, O. Maeso, Kinematic interaction factors of deep foundations with inclined piles, Earthquake Engineering \& Structural Dynamics 43 (13) (2014) 20352050 .

[14] C. Medina, J. J. Aznárez, L. A. Padrón, O. Maeso, Effects of soil-structure interaction on the dynamic properties and seismic response of piled structures, Soil Dynamics and Earthquake Engineering 53 (2013) 160-175.

[15] A. S. Veletsos, J. W. Meek, Dynamic behaviour of building-foundation systems, Earthquake Engineering \& Structural Dynamics 3 (1974) 121-138.

[16] A. S. Veletsos, V. V. D. Nair, Seismic interaction of structures on hysteretic foundations, Journal of the Structural Division, ASCE 101 (1974) 109-129.

[17] J. Avilés, L. E. Pérez-Rocha, Evaluation of interaction effects on the system period and the system damping due to foundation embedment and layer depth, Soil Dynamics and Earthquake Engineering 15 (1996) 11-27.

[18] J. Avilés, L. E. Pérez-Rocha, Effects of foundation embedment during building-soil interaction, Earthquake Engineering \& Structural Dynamics 27 (1998) 1523-1540.

[19] J. D. Achenbach. 1973. Wave propagation in elastic solids North-Holland: Amsterdam.

[20] A. C. Eringen, E. S. Suhubi. 1975. Elastodynamics, volume 2 - Linear Theory Academic Press: NY.

[21] A. K. Chopra. 2001. Dynamic of structures. Theory and applications to earthquake engineering NJ: Prentice-Hall. 